\title{
BMJ Open Protective and resilience factors to promote mental health among Indigenous youth in Canada: a scoping review protocol
}

\author{
Leah Carrier, ${ }^{1}$ Hwayeon Danielle Shin (D) ,2 Melissa A Rothfus, ${ }^{3}$ \\ Janet A Curran (iD) ${ }^{1,4}$
}

To cite: Carrier L, Shin HD, Rothfus MA, et al. Protective and resilience factors to promote mental health among Indigenous youth in Canada: a scoping review protocol. BMJ Open 2022;12:e049285. doi:10.1136/ bmjopen-2021-049285

- Prepublication history and additional supplemental material for this paper are available online. To view these files, please visit the journal online (http://dx.doi.org/10.1136/ bmjopen-2021-049285).

Received 20 January 2021 Accepted 02 November 2021

D) Check for updates

(c) Author(s) (or their employer(s)) 2022. Re-use permitted under CC BY-NC. No commercial re-use. See rights and permissions. Published by BMJ.

${ }^{1}$ Nursing, Dalhousie University, Halifax, Nova Scotia, Canada ${ }^{2}$ Institute of Health Policy, Management and Evaluation, University of Toronto, Toronto, Ontario, Canada

${ }^{3}$ W.K. Kellogg Health Sciences Library, Dalhousie University, Halifax, Nova Scotia, Canada ${ }^{4}$ Pediatrics, IWK Health Centre, Halifax, Nova Scotia, Canada

Correspondence to

Ms Hwayeon Danielle Shin;

hdanielle.shin@mail.utoronto.ca

\section{ABSTRACT}

Introduction Many mental health concerns emerge in adolescence and young adulthood, making this a critical period to initiate early interventions for mental health promotion and illness prevention. Although Indigenous young people in Canada are at a higher risk of mental health outcomes and faced with limited access to appropriate care and resources, they have unique strengths and resilience that promote mental health and wellness. Furthermore, resilience has been described as a 'healing journey' by Indigenous peoples, and interventions that account for the culture of these groups show promise in promoting mental health and wellness. As such, there is a need for innovative mental health interventions for Indigenous youth that transcend the Western biomedical model, use a strengths-based approach, and account for the cultural practices and belief systems of Indigenous peoples. This scoping review aims to explore the resilience and protective factors that promote mental health and wellness for Indigenous youth in present-day Canada with the aim of compiling and summarising the available literature on this topic to date.

Methods and analysis The review will follow Joanna Briggs Institute methodology for conducting scoping reviews. The reporting will follow the Preferred Reporting Items for Systematic Reviews and Meta-analyses Extension for Scoping Reviews checklist and guidelines. We will include both published and unpublished grey literature and search the following databases: PubMed, CINAHL, PsycINF0, Education Resources Information Center, Embase and Scopus. The search of all databases was conducted on 26 August 2021. Further, we will use government and relevant Indigenous organisation websites. Two reviewers will independently screen and select the articles and extract the data.

Ethics and dissemination No ethical approval is required for this study. We will share the results through conference presentations and an open-access publication in a peerreviewed journal. A lay-language report will be created and disseminated to community organisations that work with Indigenous youth.

\section{INTRODUCTION}

Although Indigenous children and youth face significant health inequities when compared
Strengths and limitations of this study

- While there are numerous studies illustrating the risk factors for mental health concerns among Indigenous youth, this is a novel and timely topic to comprehensively identify the range of resilience and protective factors that promote mental health and wellness.

- The study selection, data extraction and charting will be performed by two independent reviewers to minimise the risk of bias or errors.

- This scoping review has limitations regarding the language and geography, and the findings of this review may be relevant in countries of a similar context.

- Since the purpose of our scoping review is to map and characterise the evidence, we will not be conducting critically appraisals to determine the quality of individual studies to assess the risk of bias.

with the rest of the Canadian population, ${ }^{12}$ they have inherent strengths and resilience that can be drawn on to promote health and wellness. A review of critiques and recommendations for interventions promoting healthy youth development suggests that there is a need to focus on reducing risk and strengthening protective factors. ${ }^{3}$ Risk factors for adverse mental health outcomes include poverty, family conflict, neglect in early childhood, and unsafe housing. ${ }^{4}$ Fortunately, protective factors can positively modify a person's response to external and environmental stressors that result in maladaptive outcomes. ${ }^{5}$ In other words, protective factors can minimise the likelihood of dysfunction and disorder in the presence of risks. Identified protective factors are, but not limited to, living in a safe community, living free of violence, supportive relationships and education. ${ }^{4}$ Protective factors are tightly connected to resilience, which is defined as one's capacity to respond and overcome risks 
in order to avoid negative outcomes. ${ }^{6} 7$ Furthermore, resilience has also been conceptualised as function of situational and contextual factors ${ }^{8}$ or a dynamic process ${ }^{9}$ that contribute to positive outcomes. A protective model of resilience explains how protective factors operate to influence the trajectory from risk exposure to adverse outcome and modify the impact of risk exposure. ${ }^{56}$ For example, a longitudinal study of homeless youth found that physical abuse was a risk factor for greater depressive symptoms. ${ }^{10}$ However, having mentors, family and friends that youth could rely on acted as a protective factor resulting in positive social support, which then lowered the risk for depressive symptoms. ${ }^{10}$ In recognition of critical role that resilience and protective factors can play, research is needed to explore these factors for effective mental health promotion.

Resilience initiatives among Indigenous populations must address the trauma and cultural loss that occurs due to colonisation. ${ }^{11-13}$ For example, the Cree have described resilience as a 'healing journey' (Isaak et al 2015) ${ }^{14}$ Resilience identified by Indigenous youth in Canada (eg, Metis and Inuit) includes, but are not limited to, coping strategies and health promoting practices that benefit youth across varying levels of adversity. ${ }^{15} 16$ Research among Indigenous youth and adults in Canada suggests that protective factors for mental wellness include culture, community and family. ${ }^{17-19}$ For example, mental health interventions that account for the culture of Indigenous peoples show particular promise, with culture acting as a protective factor, because cultural identity is foundational to holistic health outcomes and is nurtured through participation in cultural activities. ${ }^{20} 21$

Mental health promotion and innovative approaches to promoting Indigenous youth mental health is a critical issue in Canada as mental health concerns are prevalent among youth, and various research has highlighted the high frequency of delayed or disrupted access to mental health services. ${ }^{22}{ }^{23}$ Indigenous young people experience higher rates mental health concerns and more limited access to appropriate care and resources when compared with their settler Canadian counterparts. ${ }^{24}$ For example, suicide is the second leading cause of death among all youth in Canada, ${ }^{25}$ which has been consistent for the past 10 years $^{26}$; this finding is even more dire when one considers that suicide rates among Indigenous youth are 4-10 times higher than the Canadian average. ${ }^{27}$ Strikingly, a recent Canadian study found that First Nations youth had an elevated risk of suicidal ideation and attempts if they had a parent or grandparent who attended residential school. ${ }^{18}$ Pain has been conceptualised from a mental and emotional perspective in research conducted with Indigenous youth in Mi'kma'ki (also known as Atlantic Canada). ${ }^{28}$ Additionally, First Nations children and youth have higher rates of acute and chronic pain, ${ }^{29-31}$ mental illness and substance use disorders. ${ }^{19}$ The intergenerational effect of residential school trauma and its impacts on multiple dimensions of mental health is stronger among younger youth, illustrating the importance of early intervention to promote mental health in this population. ${ }^{17}{ }^{18}$ Children who have mental health problems are more likely to experience continued challenges to mental health problems in adolescence and adulthood. ${ }^{32}$ Moreover, many mental health disorders have their onset in adolescence and young adulthood, ${ }^{33}$ making this a critical period to initiate early interventions for mental health promotion and illness prevention. ${ }^{34}$

There is a demonstrated need for innovative mental health interventions for Indigenous youth that transcend the Western biomedical model, use a strengths-based approach, and account for the cultural practices and belief systems of Indigenous peoples. ${ }^{16} 35$ A systematic review done by Rowhani and Hatala ${ }^{16}$ included 17 peer-reviewed articles and investigated how resilience of Indigenous youth is referenced in the literature and implemented in Canada. They found that these 17 articles commonly included a collaborative research approach and demonstrated diversity in culture, language and environment across the Indigenous communities in Canada. ${ }^{16}$ Furthermore, major factors contributing to resilience among these groups included cultural continuity, community and family ties and empowerment. ${ }^{16}$ Building on this previous work and specifically adding in the aspects of mental health and wellness, this current scoping review's primary objective is to explore how existing literature identifies resilience or protective factors that promote mental health and mental wellness among Indigenous youth in Canada. Furthermore, we hope to include all types of evidence that pertains to resilience and protective factors for Indigenous youth mental health. This holistic understanding of evidence is particularly useful in the current scoping review topic because Indigenous knowledge also exists in the form of grey literature. It is common for Indigenous writers to use personal narratives in describing a 'healing journey'. ${ }^{14}$ For example, community knowledge related to mental health can differ from Western understandings of individual protective or resiliency factors, with a critical lens focused on the systems and structural processes that affect the health of Indigenous youth. ${ }^{36}$ While Western literature may focus on the individual factors that can be strengthened or supported with mental health interventions, it is important to reflect on whether this is the best place to focus efforts when there are structural barriers to health equity for Indigenous youth that need to be addressed. ${ }^{36}$ Therefore, we will be including diverse evidence that systematic reviews would traditionally exclude, in addition to the published work about Indigenous knowledge in this area.

It is important to note that research has been historically conducted on Indigenous Peoples rather than in collaboration with them. As a result, there has previously been a failure to prioritise cultural conceptualisations and appropriate portrayals of Indigenous peoples, as reflected in much of the published literature. This has resulted in detrimental practices associated with the ownership, utilisation and understanding of Indigenous knowledge. ${ }^{37}$ However, it is important to note that there 
is a growing body of literature from research done in collaboration with and led by Indigenous communities in recent years. ${ }^{36}{ }^{38-42}$ For example, Kirmayer et $a \hat{l}^{36}$ discuss the concept of community resilience and how this aligns with Indigenous values and beliefs about how a person is interconnected with their environment and other people. This work defines the concept of community resilience, which may look at how individuals overcome challenges with the help of social or cultural resources in their communities. Community resilience may also refer to how communities as a whole demonstrate resiliency in the face of challenges. In another paper, Kirmayer et al describe findings from resilience research with Inuit, Metis, Mi'kmaq and Mohawk communities, describing resilience sources as a dynamic process that comes from relationships and interactions between people and their communities and social systems. ${ }^{38}$ Recognising that community involvement and priorities are critical for research related to Indigenous health and that community conceptualisations of resilience and mental health promotion may differ from Western conceptualisations, the secondary objective of this review is to identify and report the level of community involvement described in the included studies that pertain to resilience or protective factors for the mental health of Indigenous youth.

A preliminary search of the JBI Database of Systematic Reviews and Implementation Reports, the Cochrane Database of Systematic Reviews, PubMed, CINAHL and Embase was conducted and no current or underway scoping or systematic reviews on the topic were identified.

\section{REVIEW QUESTIONS}

1. What does the literature identify as resilience or protective factors for the mental health and well-being of Indigenous youth (ages 10-25) in Canada?

a. What is the level of community involvement reported in the included studies and how is community involvement described?

\section{INCLUSION CRITERIA \\ Participants}

The review will consider studies that include self-identified Indigenous youth in Canada who are between the ages of 10 and 25 years. This age bracket is consistent with how many Indigenous communities define 'youth' and with definitions used in current mental health literature. ${ }^{43} 44$ Articles will be included where participants have an official mental health diagnoses (DSM-5), ${ }^{45}$ self-identified mental health and wellness concerns, symptoms of psychological distress and/or are involved in mental health promotion and illness prevention initiatives. All genders will be included.

\section{Concept}

This scoping review will include all articles that examine resilience and/or protective factors for mental health

\begin{tabular}{ll}
\hline Table 1 & Operational terms and definitions \\
\hline Term & Definition \\
\hline $\begin{array}{l}\text { Protective } \\
\text { factors }\end{array}$ & $\begin{array}{l}\text { 'Influences that modify, ameliorate or alter a person's } \\
\text { response to some environmental hazard that } \\
\text { predisposes to a maladaptive outcome' (Rutter, p600). }\end{array}$ \\
Resilience & $\begin{array}{l}\text { Personality traits, } \\
{ }^{56} \text { function of situational and } \\
\text { contextual factors } \\
\text { or a dynamic process }\end{array}$ \\
& health outcomes.
\end{tabular}

and mental wellness among Indigenous youth. Articles that examine prevalence and incidence of mental health concerns without discussing resilience or protective factors will be excluded. For this review, the following operational terms and definitions will be used (table 1). Articles may not explicitly use the terms, 'resilience' or 'protective factors'. As such, this review will include articles if they discuss other mental health promoting factors and psychosocial factors that contribute to adaptive outcomes for mental health and wellness, all of which fall under the operationalised definitions for resilience or protective factors.

\section{Context}

This review will consider articles that are conducted in the healthcare, education or community settings in Canada. International articles where the data related to Indigenous youth in Canada can be extracted will also be included. Settings may be urban, rural or remote. Articles with Indigenous communities or Indigenous participants who live in settler communities will be included. Articles will be excluded when Canadian data is not clearly identified or able to be extracted.

\section{Types of sources}

This scoping review will consider both quantitative and qualitative designs, as well as those studies and articles that use Indigenous ways of knowing. Quantitative designs include any experimental and quasi-experimental study designs including randomised controlled trials, non-randomised controlled trials, before and after studies and interrupted time-series studies. In addition, analytical observational studies including prospective and retrospective cohort studies, case-control studies and analytical cross-sectional studies will be considered for inclusion. This review will also consider descriptive observational study designs including case series, individual case reports and descriptive cross-sectional studies for inclusion. Qualitative studies will also be considered that focus on qualitative data including, but not limited to, designs such as phenomenology, grounded theory, ethnography, qualitative description, action research and feminist research. In addition, scoping and systematic reviews that meet the inclusion criteria will also be considered and their references lists will be reviewed for primary articles. Text and opinion papers will also be considered 
for inclusion in this scoping review. Grey literature will also be considered, and may include papers, reports or policy documents related to youth mental health promotion and protective factors. Studies in English will be included. No parameters around dates will be applied.

\section{METHODS}

Scoping reviews determine the scope of existing evidence on a specific topic and identify key concepts and research gaps. ${ }^{46}$ The findings of the reviews can be used to inform future research that is focused on important knowledge gaps. ${ }^{48}$ The findings can also provide critical information for decision makers, policy-makers and clinicians in the area of Indigenous youth mental health. Therefore, a scoping review is an appropriate methodology to systematically explore all literature including peer-reviewed articles and grey literature about resilience and protective factors for Indigenous youth's mental health. To ensure replicability and rigorous methodology, the proposed scoping review will strictly adhere to the Joanna Briggs Institute methodology for scoping reviews. ${ }^{49}$ The Preferred Reporting Items for Systematic Reviews and Meta-Analyses extension for scoping reviews (PRISMA) checklist will be used to guide the reporting of this scoping review. ${ }^{51}$ Major steps comprised in our scoping review methods are: (1) identifying the research question; (2) searching for relevant studies; (3) screening and selecting relevant studies; (4) charting data and (5) summarising and presenting key findings.

\section{Search strategy}

The final search strategy and data search was developed in consultation with a JBI-trained librarian scientist. A preliminary search of PubMed and Google Scholar was undertaken to identify target articles on the topic. The text words contained in the titles and abstracts of relevant articles, the index terms, headings and MESH terms used to describe the articles were used to develop the final search strategy for PubMed. The search strategy, including all identified keywords, headings, MESH terms and index terms, was adapted for each included information source. The final search strategies conducted on 26 August 2021 for all included databases can be found in online supplemental Appendix 1. A second librarian will complete a peer review of the final search strategies using the Peer Review of Electronic Search Strategies guidelines. $^{52}$

\section{Information sources}

PubMed, Cumulative Index of Nursing and Allied Health Literature, Education Resources Information Center, Embase, PsycINFO and Scopus will be included as information sources. The search for unpublished literature will include: Grey Literature Report, conference abstracts and presentations and dissertations, government documents and relevant Indigenous organisation websites including, but not limited to Native Women's Association of Canada,
Assembly of First Nations, and the First Nations Inuit Governance Centre. Lastly, a hand search of three high impact journals (Canadian Journal of Community Mental Health, Journal of Psychiatric and Mental Health Nursing and International Journal of Mental Health Nursing) will be carried out.

\section{Study selection}

Following the search, all identified citations will be collated and uploaded into Covidence, an online software program, ${ }^{53}$ and duplicates will be removed. Titles and abstracts will then be screened by two independent reviewers for assessment against the inclusion criteria for the review. Potentially relevant studies will be retrieved in full and their citation details imported into Covidence. The full text of selected citations will be assessed in detail against the inclusion criteria by two independent reviewers. Reasons for exclusion of full text studies that do not meet the inclusion criteria will be recorded and reported in the full scoping review. Any discrepancies between the reviewers at each stage of the study selection process will be resolved through discussion, or with a third reviewer. Scoping reviews do not require methodological assessment, ${ }^{49}$ so critical appraisal will not be conducted. The results of the search will be reported and presented in a PRISMA 2020 flow diagram. ${ }^{54}$

\section{Data charting}

A draft of the data extraction instrument (online supplemental Appendix 2) has been developed by the reviewers to extract the following study information:

- Authors

- Year of publication.

- Aim of study.

- Type of study.

- Literature type.

- Country of publication.

- Study population (participants).

- Geographical setting.

- Type of service (healthcare, education, community, etc).

- Description of community involvement.

- Involved community, knowledge users or stakeholders.

- Type of mental health concerns.

- Outcome measurement tool.

- Methodology/methods.

- Use of a theory or framework.

- Definition of resilience/protective factors/coping skills used.

- Description of resilience or protective factors (this can also include other mental health promoting factors or psychosocial factors that contribute to adaptive outcomes for mental health and wellness).

- Reported outcomes.

- Study main findings and conclusion.

- Study future implications.

Two reviewers will independently extract data using the developed data extraction tool. Reviewers will meet after 
data extraction has been completed for the first three studies to identify any discrepancies and ensure consistency. The draft data extraction tool will be modified and revised as necessary during the process of extracting data from each included study. Modifications and revisions will be detailed in the full scoping review report. After finalising data extraction tool, the remaining data will be extracted by two independent reviewers. Any conflicts in the data extraction will be resolved through discussion, or with a third reviewer. Authors of papers will be contacted to request missing or additional data, where required.

Drawing on Bronfenbrenner's ecological systems theory ${ }^{55}$ as well as Indigenous knowledge about the interacting systems that influence behaviour, narrative descriptions of resilience and protective factors will be mapped on the appropriate level of ecological system (individual, family, community, cultural and systems level). Second, community involvement described in the identified citations will be characterised appropriately. As data analysis is expected to be an iterative process, necessary changes will be made and reported in the full review.

\section{Data presentation}

The extracted data will be, where appropriate, presented in a tabular form with the narratives in a manner that aligns with the objective of this scoping review. The tabular summary will highlight the major categories of the data extraction form as described above. A narrative summary will accompany the tabulated and/or charted results and will describe how the results relate to the reviews objective and questions.

A narrative description of protective factors identified by the studies and community definitions of protective factors will be provided to discuss apparent gaps in current knowledge. The use of theory and framework to understand the protective factors for Indigenous communities will also be reported, which will help illuminate contextual factors that may influence the use and implementation of future interventions in this area.

\section{Patient and public involvement}

Patients or the public were not involved in this scoping review design, including data collection and interpretation planning. However, a lay-language report will be created and disseminated to community organisations that work with Indigenous youth.

\section{Ethics and dissemination}

This is a scoping review on existing literature, and therefore, no ethical approval is required for this study. The goal of this scoping review is to map and characterise the evidence related to resilience and protective factors for mental health and wellness among Indigenous Youth in Canada. The findings of the scoping review may inform future research directions as well as mental health intervention designs that account for the cultural and belief systems of Indigenous peoples. As such, not only we will share the results through conference presentations and an open-access publication in a peer-reviewed journal but also share with key academic and community organisations (eg, Indigenous Health Interest Group at Dalhousie University, Friendship Centres, community health centres) and health systems through lay-language summaries and informal presentations.

Acknowledgements We would also like to thank Dr. Margot Latimer for reviewing an earlier draft of this protocol

Contributors LC and HDS designed the scoping review protocol including data collection and interpretation planning and drafted and revised the protocol for intellectual content. LC, HDS, MR and JAC made a final approval for the submission of the protocol. LC and HDS made an equal contribution to the manuscript and claim first coauthorships.

Funding This research received no specific grant from any funding agency in the public, commercial or not-for-profit sector.The authors have not declared a specific grant for this research from any funding agency in the public, commercial or notfor-profit sectors.

Disclaimer There are no conflicts of interest to declare with this submission.

Competing interests None declared.

Patient consent for publication Not applicable.

Provenance and peer review Not commissioned; externally peer reviewed.

Supplemental material This content has been supplied by the author(s). It has not been vetted by BMJ Publishing Group Limited (BMJ) and may not have been peer-reviewed. Any opinions or recommendations discussed are solely those of the author(s) and are not endorsed by BMJ. BMJ disclaims all liability and responsibility arising from any reliance placed on the content. Where the content includes any translated material, BMJ does not warrant the accuracy and reliability of the translations (including but not limited to local regulations, clinical guidelines, terminology, drug names and drug dosages), and is not responsible for any error and/or omissions arising from translation and adaptation or otherwise.

Open access This is an open access article distributed in accordance with the Creative Commons Attribution Non Commercial (CC BY-NC 4.0) license, which permits others to distribute, remix, adapt, build upon this work non-commercially, and license their derivative works on different terms, provided the original work is properly cited, appropriate credit is given, any changes made indicated, and the use is non-commercial. See: http://creativecommons.org/licenses/by-nc/4.0/.

ORCID iDs

Hwayeon Danielle Shin http://orcid.org/0000-0003-4037-4464

Janet A Curran http://orcid.org/0000-0001-9977-0467

\section{REFERENCES}

1 Smylie J, Fell D, Ohlsson A. Joint Working group on First Nations, Inuit, and Métis infant mortality of the Canadian perinatal surveillance system. A review of Aboriginal infant mortality rates in Canada: striking and persistent Aboriginal/Non-Aboriginal inequities. Can J Public Health 2010;101:143-8.

2 Peters PA, Oliver LN, Kohen DE. Mortality among children and youth in high-percentage First Nations identity areas, 2000-2002 and 20052007. Rural Remote Health 2013;13:2424.

3 Catalano RF, Hawkins JD, Berglund ML, et al. Prevention science and positive youth development: competitive or cooperative frameworks? $J$ Adolesc Health 2002;31:230-9.

4 Government of Canada. Protective and risk factors for mental health, 2019. Available: https://www.canada.ca/en/public-health/services/ protective-risk-factors-mental-health.html

5 Rutter M. Resilience in the face of adversity. protective factors and resistance to psychiatric disorder. Br J Psychiatry 1985;147:598-611.

6 Rutter M. Psychosocial resilience and protective mechanisms. Am J Orthopsychiatry 1987;57:316-31 10.1111/j.1939-0025.1987. tb03541.x

7 Rak CF, Patterson LE. Promoting resilience in at-risk children. $J$ Couns Dev 1996;74:368-73.

8 Masten AS, Best KM, Garmezy N. Resilience and development: contributions from the study of children who overcome adversity. Dev Psychopathol 1990;2:425-44. 
9 Luthar SS, Cicchetti D, Becker B. The construct of resilience: a critical evaluation and guidelines for future work. Child Dev 2000;71:543-62.

10 Tyler KA, Schmitz RM, Ray CM. Role of social environmental protective factors on anxiety and depressive symptoms among midwestern homeless youth. J Res Adolesc 2018;28:199-210.

11 Brooks CM, Daschuk MD, Poudrier J, et al. First Nations youth redefine resilience: listening to artistic productions of 'Thug Life' and hip-hop. J Youth Stud 2015;18:706-25.

12 Goulet L, Linds W, Episkenew J-A. Creating a space for decolonization: health through theatre with Indigenous youth. Native Stud Rev 2011;20:89-116.

13 Tousignant M, Sioui N. Resilience and Aboriginal communities in crisis: theory and interventions. J Aborig Health 2009;5:43.

14 Isaak CA, Stewart DE, Mota NP, et al. Surviving, healing and moving forward: journeys towards resilience among Canadian Cree adults. Int J Soc Psychiatry 2015;61:788-95.

15 Kral MJ, Salusky I, Inuksuk P, et al. Tunngajuq: stress and resilience among Inuit youth in Nunavut, Canada. Transcult Psychiatry 2014;51:673-92.

16 Rowhani M, Hatala A. A systematic review of resilience research among Indigenous youth in contemporary Canadian contexts. The International Journal of Health, Wellness, and Society 2017;7:45-58.

17 Bombay A, Matheson K, Anisman H. Decomposing identity: differential relationships between several aspects of ethnic identity and the negative effects of perceived discrimination among First Nations adults in Canada. Cultur Divers Ethnic Minor Psychol 2010;16:507-16.

18 Bombay A, McQuaid RJ, Schwartz F, et al. Suicidal thoughts and attempts in First Nations communities: links to parental Indian residential school attendance across development. J Dev Orig Health Dis 2019;10:123-31.

19 Health Canada and Assembly of First Nations. First Nations Mental Wellness Continuum Framework. Ottawa: Health Canada, 2015. https://thunderbirdpf.org/wp-content/uploads/2015/01/24-14-1273FN-Mental-Wellness-Framework-EN05 low.pdf

20 Reyhner J. Indigenous language immersion schools for strong Indigenous identities. Herit Lang J 2010;7:138-52.

21 LaFromboise TD, Hoyt DR, Oliver L, et al. Family, community, and school influences on resilience among American Indian adolescents in the upper Midwest. J Community Psychol 2006;34:193-209.

22 Gravel R, Béland Y. The Canadian community health survey: mental health and well-being. Can J Psychiatry 2005;50:573-9.

23 Canadian Mental Health Association. Fast Facts about Mental IIIness - CMHA National, 2020. Available: https://cmha.ca/fast-facts-aboutmental-illness

24 Canada S. Aboriginal peoples in Canada: First Nations people, Metis and Inuit: National household survey, 2011, 2013. Available: https:// www150.statcan.gc.ca/n1/daily-quotidien/130508/dq130508a-eng. htm [Accessed January 18, 2021].

25 Kutcher SP, Szumilas M. Youth suicide prevention. Can Med Assoc J 2008; 178:282-5.

26 Public health agency of Canada. Suicide in Canada: key statistics (infographic) government of Canada, 2019. Available: https://www. canada.ca/en/public-health/services/publications/healthy-living/ suicide-canada-key-statistics-infographic.html

27 Government of Canada SC. Suicide among First Nations people, Métis and Inuit (2011-2016): findings from the 2011 Canadian census health and environment cohort (CanCHEC), 2019. Available: https:// www150.statcan.gc.ca/n1/pub/99-011-x/99-011-x2019001-eng.htm [Accessed April 25, 2021].

28 Latimer M, Sylliboy JR, Francis J, et al. Co-creating better healthcare experiences for First Nations children and youth: The FIRST approach emerges from Two-Eyed s eeing. Paediatric and Neo Pain 2020;2:104-12.

29 Latimer M, Simandl D, Finley A, et al. Understanding the Impact of the Pain Experience on Aboriginal Children's Wellbeing: Viewing through a Two-Eyed Seeing Lens. First Peoples Child Fam Rev 2014:9:22-37.

30 Latimer M, Rudderham S, Lethbridge L, et al. Occurrence of and referral to specialists for pain-related diagnoses in First Nations and non-First nations children and youth. CMAJ 2018;190:E1434-40.

31 Latimer M, Sylliboy JR, MacLeod E, et al. Creating a safe space for First Nations youth to share their pain. Pain Rep 2018;3:e682 https://
journals.Iww.com/painrpts/Fulltext/2018/09001/Creating_a_safe_ space for First Nations youth to.6.aspx

32 Smetanin P, Stiff D, Briante C. The life and economic impact of major mental illnesses in Canada: 2011 to 20412011.

33 Jones PB. Adult mental health disorders and their age at onset. $\mathrm{Br} \mathrm{J}$ Psychiatry 2013;202:s5-10.

34 Mental health Commission of Canada. Changing directions, changing lives: Thethe mental health strategy for Canada, 2012 Available: https://www.mentalhealthcommission.ca/sites/default/ files/MHStrategy_Strategy_ENG.pdf

35 Sasakamoose J, Scerbe A, Wenaus I. First Nation and Métis youth perspectives of health: an Indigenous qualitative inquiry. Qual Inq 2016;22:636-50.

36 Kirmayer LJ MD, FRCPC SM, PhD WR. Metaphors and measures. J Aborig Health 2009;5:62-117.

37 First Nations Information Governance Centre. Ownership, control, access and possession (OCAPTM): the path to First Nations information governance, 2014. Available: http://books.scholarsportal. info/en/read?id=/ebooks/ebooks4/cpdc4/2018-12-11/3/10095457 [Accessed August 2, 2021].

38 Kirmayer LJ, Dandeneau S, Marshall E, et al. Rethinking resilience from Indigenous perspectives. Can J Psychiatry 2011;56:84-91.

39 Andersson N, Ledogar RJ. The CIET Aboriginal youth resilience studies: 14 years of capacity building and methods development in Canada. Pimatisiwin 2008;6:65-88.

40 PhD TM, PhD SN. Resilience and Aboriginal communities in crisis: theory and interventions. J Aborig Health 2009;5:43-61.

41 Njeze C, Bird-Naytowhow K, Pearl T, et al. Intersectionality of resilience: a Strengths-Based case study approach with Indigenous youth in an urban Canadian context. Qual Health Res 2020;30:2001-18.

42 Isbister-Bear O, Hatala A, Sjoblom E. Strengthening Âhkamêyimo among Indigenous youth: The social determinants of health, justice, and resilience in Canada's north. J Indig Well-Being 2017;2:76-89.

43 World Health Organization. Atlas : child and adolescent mental health resources : global concerns: implications for the future. Child Adolesc Ment Health Atlas 2005 https://apps.who.int/iris/handle/ 10665/43307

44 Malla A, Shah J, lyer S, et al. Youth mental health should be a top priority for health care in Canada. Can J Psychiatry 2018;63:216-22.

45 American Psychiatric Association. DSM-5 Task Force. Diagnostic and Statistical Manual of Mental Disorders : DSM-5. American Psychiatric Association 2013.

46 Armstrong R, Hall BJ, Doyle J, et al. 'Scoping the scope' of a cochrane review. J Public Health 2011;33:147-50.

47 Arksey H, O'Malley L. Scoping studies: towards a methodological framework. Int J Soc Res Methodol 2005;8:19-32.

48 Wickremasinghe D, Kuruvilla S, Mays N, et al. Taking knowledge users' knowledge needs into account in health: an evidence synthesis framework. Health Policy Plan 2016;31:527-37.

49 Peters MDJ, Marnie C, Tricco AC, et al. Updated methodological guidance for the conduct of scoping reviews. JBI Evid Synth 2020;18:2119-26.

50 Peters M, Godfrey C, Mclnerney P. Chapter 11: Scoping Reviews. In: Aromataris E, Munn Z, eds. Joanna Briggs Institute Reviewer's Manual. The Joanna Briggs Institute, 2017. https://reviewersmanual. joannabriggs.org/

51 Tricco AC, Lillie E, Zarin W, et al. PRISMA extension for scoping reviews (PRISMA-ScR): checklist and explanation. Ann Intern Med 2018;169:467-73.

52 McGowan J, Sampson M, Salzwedel DM, et al. PRESS Peer Review of Electronic Search Strategies: 2015 Guideline Statement. J Clin Epidemiol 2016;75:40-6.

53 Covidence systematic review software, 2019. Available: www. covidence.org

54 Page MJ, McKenzie JE, Bossuyt PM, et al. The PRISMA 2020 statement: an updated guideline for reporting systematic reviews. BMJ 2021;372:n71.

55 Bronfenbrenner U. Ecological models of human development. In: The International encyclopedia of education. 3. Oxford: Elsevier, 1994

56 Friborg O, Barlaug D, Martinussen M, et al. Resilience in relation to personality and intelligence. Int $J$ Methods Psychiatr Res 2005;14:29-42. 